\title{
HOSPITAL SAN JUAN DE DIOS: ACTOR Y VÍCTIMA DE LAS POLÍTICAS PÚBLICAS EN COLOMBIA
}

\begin{abstract}
María Y. Pinilla ${ }^{1, a, c}$, César E. Abadía $2, b, d$
\section{RESUMEN}

En este artículo narramos y analizamos la construcción histórica que un grupo de trabajadoras y un colectivo de organizaciones sociales hicieron del Hospital San Juan de Dios (HSJD) e Instituto Materno Infantil (IMI) en Bogotá, Colombia, en medio de la crisis neoliberal en salud. Nuestra investigación etnográfica liga las tradiciones Latinoamericanas en etnografía colaborativa y antropología histórica y fue llevada a cabo en dos espacios. En el primero de 2005-2015 realizamos conversatorios informales, talleres y entrevistas semiestructuradas con trabajadoras y trabajadores del IMI. El segundo se desarrolló en el marco de las deliberaciones de la Mesa Jurídica por el San Juan de Dios (20082009), cuyo objetivo fue instaurar una acción popular en defensa de los hospitales. Encontramos que trabajadoras y colectivos sociales se apropiaron del origen colonial de los hospitales y su institucionalización como centros de políticas de bienestar del país para resaltar su importancia patrimonial, histórica, educativa y social. Esta construcción histórica realizada por las trabajadoras critica los esfuerzos por negar o transformar el carácter público de los hospitales y sirve para instaurar distintas acciones que denuncian la reforma neoliberal en salud como la causante de la mayor crisis de los hospitales y su cierre. Las y los actores denuncian el cambio en la relación hospitales-Estado, la cual pasó de ser central para el desarrollo de las políticas sociales a una eliminación simbólica y material que favorece los intereses de mercado establecidos con el modelo neoliberal.
\end{abstract}

Palabras clave: Hospitales universitarios; Antropología cultural; Historia; Salud pública (fuente: DeCS BIREME).

\section{HOSPITAL SAN JUAN DE DIOS: ACTOR AND VICTIM OF THE PUBLIC POLICIES IN COLOMBIA}

\begin{abstract}
In this article, we narrate and analyze the historical configuration that a group of female workers and a collective of social organizations made about the Hospital San Juan de Dios (HSJD) and Instituto Materno Infantil (IMI) in Bogotá, Colombia, within the neoliberal crisis in health. Our ethnographic research intersects the Latinamerican traditions of collaborative ethnography and historic anthropology. The research was conducted in two sites. In the first one, from 2005 until 2015 , we had informal conversations and conducted workshops and semi-structured interviews with IMI workers. The second site corresponds to our participation in the deliberations of the Mesa Jurídica por el San Juan de Dios (2008-2009), which aimed to elevate a class action to defend the hospitals. We found that workers and social organizations made use of the colonial origin of the hospitals and their institutionalization as center of welfare policies in the country as a way to highlight their patrimonial, historical, educational and social importance. This historical construction critiques efforts that negate or transform the public character of the hospitals and helped them carry on different actions to denounce the neoliberal health care reform as the cause of the hospitals most important crisis and closing. The different actors denounce the change in the hospitals-state relationship, which transited from being central for the development of social policies to reflecting a symbolic and material elimination of the hospitals. Such transition benefits the market interests established by the neoliberal model
\end{abstract}

Key words: Hospitals, University; Anthropology, cultural; History; Public Health (source: MeSH NLM).

Esmeralda mira al Hospital San Juan de Dios (HSJD) desde uno de los grandes ventanales del Hospital Materno Infantil (IMI) y se pregunta: "¿cómo es posible que este país haya dejado cerrar estos hospitales?" Meses más tarde -en el año 2006-, Ángela, secretaria del Materno, narra cómo el hospital entró en crisis financiera.
Cuenta que sus trabajadoras ya habían acumulado varios meses sin recibir salarios y se había iniciado el proceso de liquidación. Aclara a su audiencia que estos hospitales siempre habían servido a las poblaciones más pobres del país desde la época colonial, y que las trabajadoras defendian a su institución, sus derechos laborales e,

\footnotetext{
Programa de Bioética, Universidad El Bosque, Bogotá, Colombia.

2 Departamento de Antropología e Instituto de Derechos Humanos, Universidad de Connecticut. Connecticut, EE.UU.

a Antropóloga; ${ }^{\mathrm{b}}$ odontólogo; ${ }^{\mathrm{c}}$ magister en Estudios de Género; ${ }^{\mathrm{d}}$ doctor en Antropología Médica.

Recibido: 19/05/2017 Aprobado: 14/06/2017 En línea: 28/06/2017
}

Citar como: Pinilla MY, Abadía CE. Hospital San Juan de Dios: actor y víctima de las políticas públicas en Colombia. Rev Peru Med Exp Salud Publica. 2017;34(2):287-92. doi: 10.17843/rpmesp.2017.342.2888 
inclusive, los bienes y propiedades de los hospitales. Con rabia y dolor contaba: Nos llegó la policía y nos llegaron con camiones y nos llegó toda la maquinaria del Estado, como si fuéramos unas delincuentes y estábamos solo mujeres... y entonces dice uno cómo el Estado nos ha abandonado...(1)

La crisis de los hospitales públicos y universitarios en Colombia, como el IMI (inicialmente especialidad del HSJD y desde mediados del siglo XX funcionando como hospital independiente) y el HSJD, se inició con la Ley 100 promulgada en el año 1993, la cual, siguiendo parámetros internacionales establecidos por el Banco Mundial, privatizó la salud y la seguridad social en el país al imponer un modelo de aseguramiento individual y universal administrado por empresas aseguradoras en salud de capital privado o mixto que fomentan la nueva cultura de la rentabilidad empresarial en salud ${ }^{(2,3)}$. Si bien se han discutido los efectos de esta reforma en términos de la consolidación de inequidades en salud, aumentos de gasto de bolsillo e incremento de la fragmentación del sistema y de las barreras de acceso económicas, geográficas y burocráticas ${ }^{(4-8)}$, es menos conocida la forma cómo los trabajadores de hospitales públicos reflexionan sobre la reforma a la salud en el país y reconstruyen su historia institucional para poder investir al hospital como "sujeto de derechos" (9) e instaurar acciones en su defensa.

En este artículo narramos y analizamos la construcción histórica que un grupo de trabajadoras y un colectivo de organizaciones sociales hicieron del HSJD y el IMI en Bogotá, Colombia, en medio de la crisis neoliberal en salud. Nuestra investigación etnográfica liga las tradiciones latinoamericanas en etnografía colaborativa, con su propuesta de dinamizar la antropología al comprometerse con las luchas políticas de los sujetos con los que interactuamos en el trabajo de campo ${ }^{(10)}$, y la antropología histórica, en la cual teorías sociales críticas son utilizadas para hacer una "lectura etnográfica" de archivos y fuentes secundarias ${ }^{(11)}$. La investigación se realizó en dos espacios; el primero, que comprendió el periodo 2005-2015 en el que se realizaron conversatorios informales, talleres y entrevistas semiestructuradas a trabajadores del IMI, y una segunda etapa, en el marco de las deliberaciones de la Mesa Jurídica por el San Juan de Dios (2008-2009), cuyo objetivo fue instaurar una "acción popular" en defensa de los hospitales. La Mesa Jurídica por el San Juan de Dios fue un espacio conformado por diversas organizaciones sociales con el objetivo de denunciar al Estado por el incumplimiento de los acuerdos de protección del HSJD.

\section{ORIGEN, AUGE, CRISIS Y LIQUIDACIÓN DE HSDJ}

Es significativo que las trabajadoras del HSJD y del IMI se convirtieron en grandes "historiadoras nativas" (9), sus pesquisas, material histórico y compromiso político forman parte de documentos oficiales, documentales y litigios jurídicos importantes para la defensa de los hospitales y de sus acreencias laborales. Por ejemplo, gracias a ellas, el Congreso de la República declaró a los hospitales monumentos nacionales y patrimonio cultural de la nación (Ley 735 de 2002); el Consejo de Estado derogó en 2005 dos decretos que habían cambiado la figura jurídica del hospital de institución pública a fundación privada, y sigue en curso una denuncia por detrimento patrimonial. Junto con ellas y los colectivos que integraron la mesa jurídica, identificamos documentos claves para discutir cómo la historia del hospital se enmarcaba en las políticas públicas del país e instaurar acciones para enfrentar la crisis ocasionada por el neoliberalismo.

\section{¿1537- 1835?: ORÍGENES DE UN CONVENTO-HOSPITAL DE CARÁCTER PÚBLICO}

En algunos estudios y presentaciones académicas, las trabajadoras señalaban que el origen del HSJD era el Hospital San Pedro, creado mediante Cédula Real de 1537 y 1542 por el rey de España Carlos V, y fundado en 1564 , cuando el arzobispo Juan de los Barrios y Toledo le otorgó predios y casas ubicadas en el centro de Bogotá. No obstante, estos predios estaban destinados a atender a los religiosos llegados de España ${ }^{(12)}$, por lo que la institución no tendría un carácter público adscrito a la corona, como las trabajadoras pensaban, sino uno de carácter personal y privado, debido a que el fundador se reservaba el derecho de habitar en sus casas hasta su muerte ${ }^{(12,13)}$. Para la historiadora Estela Restrepo, el hospital tiene su origen en 1635, cuando se inaugura con diecisiete camas bajo la dirección de la orden de San Juan de Dios ${ }^{(12)}$. Desde entonces y hasta 1835 el hospital será una obra pía, es decir, una casa de redención de legados y caudales que permitían la manutención de la obra, en donde se celebraban misas de por vida por el donante. Frente a los debates sobre el origen de los hospitales, que define su figura jurídica y las deudas por acreencias laborales en disputa, las trabajadoras pidieron un pronunciamiento del Consejo de Estado. Finalmente, en sentencia del 8 de marzo de 2005, este ente aclaró que el origen jurídico del HSJD es de 1723, y le asigna un carácter público.

La administración del hospital en la colonia y la naciente república a inicios del siglo XIX pasaría por varias instituciones administrativas de la Corona, la Iglesia española y el Estado (14). Las trabajadoras siempre resaltaron que el hospital, desde sus inicios, era una institución fuerte desde el punto de vista económico al poseer entradas por concepto de renta urbana derivada de sus múltiples propiedades y terrenos, los capitales puestos a censos, la participación en los diezmos de la región, los aportes reales y, finalmente, el quinto que proviene de la 
obligación de admitir en el hospital a soldados ${ }^{(14)}$. Para principios del siglo XIX, la economía del hospital declinó, al parecer por la gran cantidad de personas que debía atender, incluyendo las múltiples y repetidas epidemias de viruela y "peste" que afectaron la capital (15). Con el fin de librar a la población de curanderos, charlatanes y sobanderos ${ }^{(16)}$ y de responder al contexto histórico, principalmente las guerras durante la independencia, se propuso incorporar distintos "avances" que se hacían en Europa, lo cual conformaría posteriormente la hegemonía de la "medicina moderna" al interior del hospital. Además, se planteó la necesidad de hacer de la medicina una práctica científica basada en la cirugía; se creó la primera facultad y se aumentó el número de camas ${ }^{(13-15,17)}$.

\section{5 -1868: DE LA CARIDAD A LA BENEFICENCIA}

La caridad se estructura desde el siglo XIX como política pública que mantiene el ordenamiento social y político colonial, en el cual se legitiman las diferencias entre individuos y grupos a partir de la raza, la moral y el linaje ${ }^{(18)}$. La iglesia católica promovió la creencia de que la pobreza, la indigencia y la enfermedad eran designios superiores sagrados y que ante el sufrimiento solo cabía la compasión. A mediados del siglo XIX, la riqueza pasó a ser sinónimo de una racionalidad civilizada y la pobreza a una racionalidad bárbara y primitiva producto de una debilidad moral y racial de los grupos subordinados, en donde la caridad solo era posible para aquellos grupos en donde la irracionalidad era irredimible, particularmente ancianos, mujeres desvalidas y niños ${ }^{(18)}$. Estos cambios sumados a una creciente valorización económica del mundo y aumento de la población marginal llevo a un quiebre paulatino de la caridad, para dar paso a la política pública de beneficencia que se estructura en saberes técnicos amparados por la filantropía del capital privado nacional como por las burocracias del Estado ${ }^{(13,18)}$. Sin embargo, los recursos dados por estos eran mínimos y solo beneficiaban a la minoritaria población de trabajadores asalariados. En este nuevo ordenamiento las comunidades marginadas quedaron rezagadas y continuarían recibiendo atención en las antiguas instancias caritativas.

En este marco, el hospital va adquiriendo paulatinamente el carácter de institución del Estado, enfrentando nuevas epidemias y atendiendo pacientes crónicos. Sin embargo, como "hospital de caridad" continuará sirviendo de asilo para achacosos, vagabundos e incurables ${ }^{(12)}$. La beneficencia a través de la filantropía, paso a ser una virtud laica, misericordia secularizada ${ }^{(13)}$.

\section{8-1959: EL HOSPITAL UNIVERSITARIO Y LAASISTENCIA EN UN SISTEMA DE SALUD FRAGMENTADO}

Al inicio de este periodo, el HSJD era denominado "hospital general" (12) pues atendía todo tipo de patologías bajo tres mentalidades: anatomoclínica, fisiopatológica y etiopatológica ${ }^{(19)}$. Adquirió un carácter civil, público y moderno desde su unión a la Facultad de Medicina de la Universidad Nacional de Colombia (UNAL), creada en 1868, la cual asumió su regencia académica. El HSJD pasó a ser administrado por la Junta General de Beneficencias, con la idea de romper los legados coloniales y constituir los principios de nación y estado modernos ${ }^{(13)}$; sin embargo, la influencia religiosa seguiría signando al hospital ${ }^{(12,13)}$.

En 1906 se propuso su ampliación para responder a la población en aumento, las deficientes condiciones de vida y la guerra civil. En 1911 la Asamblea de Cundinamarca autorizó a la Junta General de Beneficencias y al gobernador gestionar la cesión gratuita del terreno Molino de la Hortúa, con el fin de iniciar la construcción de la nueva sede del HSJD, la cual se inició en 1924 , con carácter oficial de asistencia pública a cargo de la Beneficencia de Cundinamarca (13), en donde permanece hasta la actualidad. En 1930, Olaya Herrera, representante del partido liberal, es elegido presidente, quebrando la hegemonía conservadora que llevaba 45 años en el poder. Planteó la necesidad de sustentar la asistencia pública sobre el principio de la obligación del Estado, lo que significó cambiar la connotación de beneficencia por asistencia; es decir, la caridad laica se transformó en una mayor responsabilidad institucional, respondiendo a los nuevos perfiles epidemiológicos del país ${ }^{(13,20)}$ y al movimiento social de la época.

Hacia mediados del siglo $\mathrm{XX}$ surgió un cambio radical en las inversiones sociales; las instituciones privadas $y$ filantrópicas poseen un interés comercial que se focaliza en los trabajadores (sector productivo), en tanto que el Estado orienta su política hacia los grupos marginales excluidos del empleo formal ${ }^{(18)}$. Esta idea es respaldada por las nacientes industrias y empresas dispuestas a racionalizar el funcionamiento y la inversión, así como por las apreciaciones de los técnicos sociales de la época, particularmente los médicos, que afirmaban que era necesaria una intervención social más decidida y menos filantrópica para atender a los pobres. Estos eventos, conjugados con las reclamaciones de los trabajadores sindicalizados de distintos sectores, convirtieron a los favores tradicionales en derechos ligados al trabajo y a las obligaciones en deberes (de empresas y el Estado), situación determinante para la política pública de la asistencia ${ }^{(21)}$.

Debido a estos ideales, la atención en salud en el país para la primera mitad del siglo $\mathrm{XX}$ transitó hacia un modelo de atención "fragmentado", constituido por tres sectores: privado, seguridad social para los trabajadores y beneficencia pública para los pobres y marginales. Los hospitales públicos como el HSJD y el IMI siguieron 
operando bajo una práctica de beneficencia dirigida a los pobres y sostenida por personas pudientes y algunos recursos del Estado ${ }^{(22)}$. La presencia religiosa se mantuvo a través de los oficios eclesiásticos y las monjas/enfermeras, quienes tenían contrato con el hospital para administrar o prestar atención en los distintos servicios.

\section{0- 1993: DESARROLLISMO Y ATENCIÓN PÚBLICA EN EL SISTEMA NACIONAL DE SALUD}

Para finales de los años 50 , los discursos de desarrollo y gestión pública buscaban que la inversión técnica y tecnológica planificada a gran escala universalizara, entre otros, a la salud ${ }^{(18)}$. Hacia los años 60 , se discutía acerca de la atención en salud como obligación del Estado y derecho social no solo ligado al trabajo. Con la creación del Sistema Nacional de Salud en 1975, el país se movió durante casi veinte años, con aciertos y desaciertos, hacia la atención primaria en salud, la descentralización y los sistemas locales de salud (23). Este nuevo ordenamiento consolidaría redes distintas de atención, una de seguridad social para trabajadores (financiada con aportes de trabajadores, empresas y el Estado) y la otra para el resto de la población (subsidiada totalmente por el Estado). Tanto la seguridad social como las redes públicas pensaban la ciudad y el país por regiones, organizadas alrededor de una clínica privada o un hospital universitario público que concentraban la atención de alto nivel de complejidad. Sin embargo, nunca se alcanzó tal nivel de organización ni de territorialización e integración por niveles de atención. En el HSJD y el IMI se atendían pacientes de varios niveles de complejidad y de otras regiones de la ciudad e incluso del país, la mayoría de manera gratuita. Sus desequilibrios económicos se relacionarían con la ausencia de políticas claras de planeación y financiación de la salud pública en el país, con la mediación administrativa por parte de la Beneficencia que actuaba como carta política y con el hecho de ser casi el único centro de urgencias de Bogotá. La voluntad de brindar una atención gratuita, universal y de alto nivel generó problemas de continuidad y suficiencia de la financiación estatal del hospital, llevando a periodos críticos de recursos desde los años sesenta.

Por medio del Decreto 1373 de 1979, el gobierno de Turbay Ayala cambió la naturaleza jurídica del HSJD y del IMI transformándolos en la Fundación San Juan de Dios, institución privada sin ánimo de lucro. En medio de las ambigüedades jurídico-administrativas, la fundación vendió varios de sus bienes y terrenos. Años más tarde, las trabajadoras aportaron pruebas para que el Consejo de Estado derogara dicho decreto y junto con la Mesa Jurídica identificaron documentos claves para señalar que la pérdida de bienes patrimoniales obedecía a un manejo "por feudos políticos" de los bienes del hospital, que se cedieron o vendieron inicialmente a entidades públicas por sumas irrisorias o "bonos" que nunca se hicieron efectivos. Las trabajadoras también identificaron con claridad a quienes, en sus palabras, "acabaron" con el hospital o se lucraron de sus riquezas.

Durante estas décadas, de la mano de la UNAL, el hospital adquirió prestigio nacional e internacional por su calidad educativa y sus avances y desarrollos técnico-científicos. Sin embargo, el problema económico seguiría siendo una constante, en tanto la atención excedía el presupuesto asignado al inicio del año, convirtiéndose en práctica común que los hospitales públicos recibieran adiciones presupuestales en el último trimestre para finalizar sus actividades de atención directa y poder pagar acreencias laborales, proveedores y servicios públicos.

\section{3 - ÉPOCA ACTUAL: PRIVATIZACIÓN DE LA SALUD Y EL FIN DE LOS HOSPITALES PÚBLICOS}

En 1993 se aprobó la Ley 100 que instaura el nuevo Sistema General de Seguridad Social en Salud. Cumpliendo los principios de la reforma neoliberal (aseguramiento individual y subsidio a la demanda), se incorporó la figura de intermediación financiera a cargo de aseguradoras públicas y privadas de servicios en salud, las cuales pasan a administrar los recursos del sistema, afiliar a los ciudadanos y pagar las cuentas a los hospitales. Esta reforma afianzó e institucionalizó desigualdades de clase social al permitir dos paquetes de servicios de salud diferenciados, uno para personas con capacidad económica llamado contributivo y el otro, con un paquete aun más reducido de servicios, para personas sin capacidad económica llamado subsidiado ${ }^{(3,7,24)}$. Nuevas instituciones de salud se unieron a las antiguas clínicas privadas para conformar las redes de servicios del sector contributivo, mientras las públicas atenderían al régimen subsidiado.

La misión y cultura institucional de los hospitales públicos sufrió cambios importantes, pasando de recibir recursos directos del Estado a someter cuentas de cobro a las aseguradoras por cada uno de los servicios prestados ${ }^{(25)}$. Así, la lógica de la facturación y manejo de cuentas se apoderó de los hospitales públicos planteando una competencia desleal con las nuevas instituciones de prestación de servicios de salud, las cuales nacían preparadas para navegar en el nuevo sistema. Gran parte de los problemas de sostenibilidad financiera de los hospitales públicos obedecían a legados administrativos, financieros y organizacionales que los hacian menos competitivos económicamente. EI HSJD y el IMI insistían en ofrecer tratamientos integrales y de alta calidad que no siempre eran pagados o cuyos pagos no 
reflejaban los gastos asociados con nóminas más grandes y con mayores beneficios laborales en comparación con la sobreexplotación que empezaron a vivir los trabajadores de la salud en otras entidades ${ }^{(4,7,8)}$. Las trabajadoras reconocen que les fue imposible negar la atención a las personas por falta de recursos o los frecuentes problemas administrativos impuestos por el nuevo sistema, intentando continuar así con la larga tradición caritativa.

Con el tiempo, se empezó a develar la práctica sistemática de negación de servicios y de secuestro de los recursos mediante las "glosas" que los auditores médicos de las aseguradoras hacían a las cuentas de las instituciones en salud, aunado a prácticas de corrupción de las aseguradoras (malversación de fondos) y competencia desleal, incluyendo la integración vertical entre aseguradoras y sus instituciones y redes de servicios ${ }^{(8,26-28)}$.

A finales de los noventa, la crisis económica, el cierre y la liquidación de hospitales públicos, empezaron a ser parte de las noticias cotidianas. Finalmente, en el 2001, el Ministerio de Salud impidió la remisión de pacientes al HSJD y la empresa distribuidora de energía de Bogotá suspendió el servicio. En el IMI, se nombró una agente liquidadora, quien arrendó el predio a otro hospital en el 2006 y empezó una práctica de aterrorizar a las trabajadoras con el fin de que renunciaran (28). Muchas de las trabajadoras firmaron renuncias "voluntarias"; aquellas que se negaron, vieron sus nombres en un edicto de diciembre de 2006 en el que se les anunciaba que su vínculo con la institución había terminado. Desde entonces, y hasta la fecha de este escrito (2017), las batallas legales de algunas trabajadoras por sus acreencias laborales continúan. Pugnas entre distintas administraciones, tanto de la Gobernación del departamento de Cundinamarca como de la Alcaldía de Bogotá en los últimos años resultaron en propuestas de cierre definitivo, venta, demolición o remodelación y reapertura, muchas de las cuales fueron bloqueadas por las trabajadoras mediante la ocupación física de los espacios o la instauración de medidas de protección ante distintos entes del Ministerio Público y la rama judicial.

Finalmente, en el 2015, la alcaldía de Bogotá logró que la Asamblea de Cundinamarca le autorizara la compra de los hospitales. El IMI y el HSJD reabrieron algunos servicios de atención clínica, pero ahora administrados por otros hospitales de la ciudad con un claro énfasis en la facturación, explotación del personal y servicios no integrales.

\section{LOS HOSPITALES Y SUS TRABAJADORAS: ACTORES Y VÍCTIMAS DE LAS POLIITICAS PÚBLICAS}

La reconstrucción histórica del origen colonial de los hospitales y su posterior institucionalización como centro de las políticas de bienestar para el Estado colombiano plantea dos grandes elementos. Por un lado, la apropiación que las trabajadoras hacen de la historia del hospital como elemento fundamental para denunciar la situación de abandono, desfinanciación y desfalco del hospital y la pérdida de su identidad y derechos laborales. Por otro lado, ellas logran ubicar al hospital dentro del vaivén político, social y económico del país para mostrar como es el modelo neoliberal el que finalmente lo destruye, acabando así con su condición de trabajadoras y una de las propuestas más importantes de salud integral y de alta calidad en la historia del país. El modelo neoliberal rompe con las ideas de gratuidad, educación y atención médica integral. Los pobres siguen siendo el centro de atención de los hospitales públicos, aunque han dejado de ser vistos como objetos de caridad para ser considerados objetos de gestión financiera del mercado de la salud. Por su parte, las trabajadoras de la salud se convierten en personal sobreexplotado debido a la flexibilización del trabajo.

Estos resultados nos permiten ver cómo el Estado en el neoliberalismo no desaparece sino que su papel cambia, de gestionar directamente a los pobres mediante caridad, beneficencia y asistencia, se convierte en un ente que permite la consolidación de políticas ancladas al mercado en salud con beneficios directos al sector empresarial y financiero. Por otro lado, la rama judicial y los entes de control público adquieren un papel fundamental, aunque limitado, en la defensa del carácter público de la salud y los derechos asociados a la salud y el trabajo.

Esta reconstrucción histórica nos permite aclarar cómo el neoliberalismo transforma o elimina instituciones con vocación de enseñanza y solidaridad con los sectores marginalizados. El HSJD y el IMI se convirtieron en amenazas al nuevo orden financiero en salud al permanecer como símbolos de la atención integral, pública y gratuita (subsidio a la oferta). Independiente de los múltiples problemas que presentaban, su insistencia en brindar atención gratuita, integral y en contextos de enseñanza médica pública resultó incómoda para un modelo basado en el mercado individual de pólizas en salud administradas por el sector financiero que necesitaba consolidarse. En este sentido, el cierre de instituciones insignias de la salud en el país y el "despido" al que fueron sometidas sus trabajadoras es uno de los resultados del modelo neoliberal.

Contribuciones de autoría: MYP y CEA han participado en la concepción del artículo, la recolección de los datos, su redacción y aprobación de la versión final.

Fuentes de financiamiento: autofinanciado.

Declaración de conflictos de interés: los autores declaran no tener conflictos de interés. 


\section{REFERENCIAS BIBLIOGRÁFICAS}

1. Abadía C, Melo MA, Pinilla MY, Ariza K, Ruíz C. Algunas violencias del capitalismo en Colombia. Dispositivos de tortura y autodestrucción. Maguaré. 2011;25(1):203-40.

2. Giraldo C.¿Protección o desprotección social? Bogotá: Ediciones Desde Abajo; 2007.

3. Hernández M. Neoliberalismo en Salud: desarrollos, supuestos y alternativas. En: Botero D, editor. La falacia neoliberal. Crítica y Alternativas. Bogotá: Universidad Nacional de Colombia; 2003. p. $347-$ 61.

4. Abadía CE, Oviedo DG. Bureaucratic itineraries in Colombia. A theoretical and methodological tool to assess managed-care health care systems. Soc Sci Med. 2009;68(6):1153-60. doi: 10.1016/j.socscimed.2008.12.049

5. Ewig C, Bello AH. Gender equity and health sector reform in Colombia: mixed state-market model yields mixed results. Soc Sci Med. 2009;68(6):1145-52. doi: 10.1016/j. socscimed.2008.12.039

6. Echeverry López ME, Arango Castrillón A, editores. Indignación justa: estudio sobre la acción de tutela en salud en Medellín. Medellín: Hombre Nuevo Editores; 2013.

7. Yepes JF, Ramírez M, Sánchez LH, Ramírez ML, Jaramillo I. Luces y sombras de la reforma de la salud en Colombia. Ley 100 de 1993. Bogotá: Mayol Ediciones; 2010.

8. Molina G, Muñóz IF, Ramírez A, editores. Dilemas en las decisiones en la atención en salud. Ética, derechos y deberes constitucionales frente a la rentabilidad financiera. Bogotá: Instituto de Estudios del Ministerio Público; 2009.

9. Góngora A, Fergusson S, Borja R, Castro M, Arias E. El San Juan muere de pie: la vida social de un hospital y la construcción de una causa [Internet]. Bogotá: Universidad Nacional de Colombia; 2013 [citado 15 may 2017]. Disponible en: http://siamisderechos. org/banco/todo/ATT1384541109.pdf

10. Rappaport J. Beyond Participant Observation: Collaborative ethnography as theoretical innovation. Collab Anthropol. 2008;1:1-31. doi: 10.1353/ cla.0.0014

11. Pineda R. La Historia, los antropólogos y la Amazonía. Antipod. Rev. Antropol. Arqueol. 2005;1:121-35.

12. Restrepo E. El Hospital San Juan de Dios 1635-1895.Una historia de enfermedad, pobreza y muerte en Bogotá. Bogotá: Universidad Nacional de Colombia; 2011.

13. Bochetti C, Arteaga JM, Palacios M. Hospital universitario: desde San Juan de Dios hasta la construcción de un nuevo proyecto. Bogotá: Universidad Nacional de Colombia; 2005.

14. Vargas J. La Sociedad de Santafé Colonial. Bogotá: CINEP; 1990.

15. Ibáñez M. Memorias para la historia de la medicina en Santa Fe. 2da ed. Bogotá: Universidad Nacional de Colombia; 1968.

16. Mejía GA. La ilustración en la Nueva Granada, una aproximación. En: Mejía GA, editor. Aspectos de la Ilustración Neogranadina. Bogotá: Fundación Editorial Argumentos; 1996.

17. Alcácer A de F, Domingo de Petrés. Misional Padres Capuchinos. Bogotá: Ediciones Manrique; 1958.

18. Serna A. Ciudadanos de la geografía tropical. Ficciones históricas de lo ciudadano. Bogotá: Universidad Distrital Francisco José de Caldas; 2006.

19. Romero M, Zambrano M, Darío M. Historia del Hospital San Juan de Dios de Bogotá. Bogotá: Alcaldía Mayor de Bogotá-Instituto Patrimonio Cultural; 2008.
20. Sánchez C. De la aldea a la metrópoli. Seis décadas de vida cotidiana en Bogotá, 1900-1959. Bogotá: IDCT. TM Editores; 1998.

21. Archila M. Cultura e Identidad Obrera, Colombia 1910-1945. Bogotá: CINEP; 1991.

22. Hernández M. La salud fragmentada en Colombia, 1910-1946. Bogotá: Universidad Nacional de Colombia; 2002.

23. Hernández M, Obregón D. La Organización Panamericana de la Salud y el estado colombiano: cien años de historia 1902-2002. Bogotá: Organización Panamericana de la Salud; 2002.

24. Gaviria A, Medina C, Mejia C. Evaluating the impact of health care reform in Colombia: from theory to practice [Internet]. Bogotá: Universidad de los Andes; 2006 [citado 15 may 2017]. Disponible en: https://core. ac.uk/download/pdf/6395237.pdf

25. Valdés E. La transformación de la gestión hospitalaria en el Distrito Capital. Pre-Til. 2008;6(17):64-84

26. Campos J, Rivera M, Castañeda MY. El Malestar de la salud en Colombia. Econ Colomb. 2004 Jul;303:8-33.

27. Robledo JE. El verdadero Ministerio de Salud es el "Cartel de las EPS". Econ Colomb. 2012;336:35-46.

28. Ardila A. Neoliberalismo y medicina general De la profesión liberal al trabajo explotado. Doctoral Thesis. Public Health. Universidad Nacional de Colombia.; 2016.

Correspondencia: César Ernesto Abadía Barrero

Dirección: 354 Mansfield Rd. Storrs, CT. USA 06269

Teléfono: (1) 6179993612

Correo electrónico:cesar_abadia@post.harvard.edu 